\title{
BMJ Open Cost-effectiveness analysis of pembrolizumab plus chemotherapy for previously untreated metastatic non- small cell lung cancer in the USA
}

\author{
Xiaohui Zeng, ${ }^{1,2}$ Xiaomin Wan, ${ }^{2,3}$ Liubao Peng, ${ }^{2,3}$ Ye Peng, ${ }^{2,3}$ Fang Ma, ${ }^{4}$ Qiao Liu, ${ }^{2,3}$ \\ Chongqing Tan (D) ${ }^{2,3}$
}

To cite: Zeng $X$, Wan $X$, Peng L, et al. Cost-effectiveness analysis of pembrolizumab plus chemotherapy for previously untreated metastatic non-small cell lung cancer in the USA. BMJ Open 2019;9:e031019. doi:10.1136/ bmjopen-2019-031019

- Prepublication history and additional material for this paper are available online. To view these files, please visit the journal online (http://dx.doi. org/10.1136/bmjopen-2019031019).

Received 11 April 2019 Accepted 22 November 2019

Check for updates

(C) Author(s) (or their employer(s)) 2019. Re-use permitted under CC BY-NC. No commercial re-use. See rights and permissions. Published by BMJ.

${ }^{1}$ PET-CT Center, The Second Xiangya Hospital of Central South University, Changsha, Hunan, China

${ }^{2}$ Institute of Clinical Pharmacy, Central South University, Changsha, Hunan, China ${ }^{3}$ Department of Pharmacy, Second Xiangya Hospital of Central South University, Changsha, Hunan, China ${ }^{4}$ Department of Oncology, The Second Xiangya Hospital of Central South University, Changsha, Hunan, China

Correspondence to Professor Chongqing Tan; tanchongqing@csu.edu.cn

\section{ABSTRACT}

Objectives Evaluating the cost-effectiveness of pembrolizumab plus standard chemotherapy in the firstline setting for patients with metastatic non-small cell lung cancer (NSCLC) from the US payer perspective.

Design A Markov model was constructed to analyse the cost-effectiveness of pembrolizumab plus chemotherapy in the first-line treatment of metastatic NSCLC. Health outcomes were estimated in quality-adjusted life-years (QALYs). The cost information was from Medicare in 2018. One-way and probabilistic sensitivity analyses examined the impact of uncertainty and assumptions on the results. Setting The US payer perspective.

Participants A hypothetical US cohort of patients with previously untreated metastatic nonsquamous NSCLC without EGFR or ALK mutations.

Interventions Pembrolizumab plus chemotherapy versus chemotherapy.

Primary outcome measures Costs, QALYs, incremental cost-effectiveness ratio (ICER) of pembrolizumab plus chemotherapy expressed as cost per QALY gained compared with chemotherapy

Results The base case analysis demonstrated that pembrolizumab plus chemotherapy provided an additional 0.78 QALYs at incremental cost of $\$ 151409$, resulting in an ICER of \$194 372/QALY. ICER for pembrolizumab plus chemotherapy was $>\$ 149680 /$ QALY in all of our univariable and probabilistic sensitivity analyses.

Conclusions Pembrolizumab in addition to chemotherapy provides modest incremental benefit at high incremental cost per QALY for the treatment of previously untreated metastatic NSCLC.

\section{INTRODUCTION}

Globally, lung cancer had an incidence rate of 27.4 per 100000 and a mortality rate of 23.1 per 100000 in $2018,{ }^{1}$ and non-small cell lung cancer (NSCLC) accounted for the vast majority of these cases. ${ }^{2}$ Multiple drug regimens are available for the treatment of advanced NSCLC, including platinumbased combination chemotherapy, anaplastic lymphoma kinase (ALK) tyrosine kinase inhibitor (TKI), epidermal growth factor
Strengths and limitations of this study

- The study strengths of this model-based economic assessment include that it is based on rigorous randomised controlled trials.

- From a US payer perspective, the cost and outcome data included in the model are collected for analysis.

- The limitation of this study is that because of the limited time scale of the model and the lack of longterm data, not all potential outcomes are included.

receptor (EGFR)TKI and immune checkpoint inhibitors. ${ }^{2}$ Immune checkpoint inhibitors showed higher efficacy and less toxicity compared with other therapies. ${ }^{3}$

A new era of treating advanced NSCLC is upon us after the emergence of immunosuppressive agents. ${ }^{4}$ Immune checkpoint inhibitors improve antitumor immunity by inhibiting programmed death 1 (PD-1) receptor or programmed cell death ligand 1 (PD-L1). ${ }^{2}$ 5-7 Pembrolizumab, a PD-1 inhibitor, was approved by the US Food and Drug Administration (FDA) for treatment of advanced NSCLC in $2015 .{ }^{8}$ The Keynote189 clinical trial showed pembrolizumab in combination with pemetrexed plus carboplatin or cisplatin could extend progressionfree survival (PFS) by 3.9 months for patients with metastatic NSCLC without sensitising ALK or EGFR mutations. ${ }^{10}$

Although pembrolizumab plus chemotherapy improved survival significantly, the additional cost was notably high. Therefore, it is worth discussing whether pembrolizumab plus chemotherapy is a cost-effective regimen. The goal of this study was to analyse the cost-effectiveness of pembrolizumab plus chemotherapy for previously untreated metastatic NSCLC without ALK or EGFR mutations from the US payer perspective. 


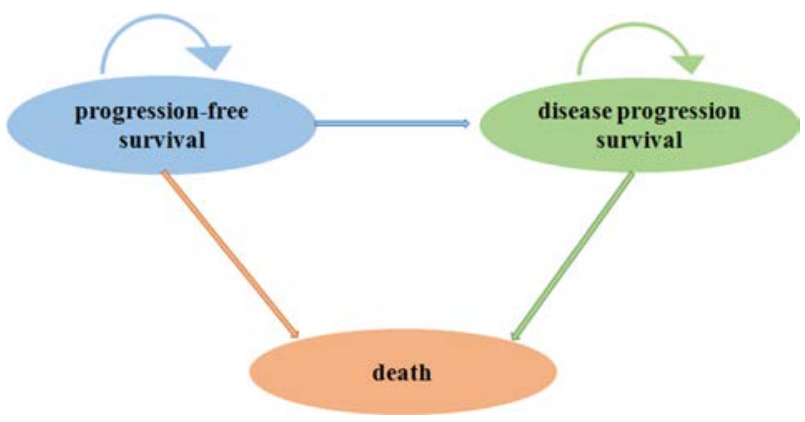

Figure 1 State transition diagram. The three circles show three main health states. Patients can transition from 'progression-free survival' to 'disease progression survival' or 'death'

\section{MATERIAL AND METHODS}

\section{Decision model}

A Markov model was built to simulate the flow process of patient morbidity, treatment and survival for previously untreated metastatic NSCLC, using three states, namely PFS state, disease progression survival state and death (figure 1). All patients entranced the model in the PFS state, with the treatment of pemetrexed combined platinum plus pembrolizumab or placebo. Patients who experienced progression could receive carboplatin plus pemetrexed, docetaxel plus ramucirumab, docetaxel monotherapy, nivolumab or pembrolizumab, because these regimens were used most in the Keynote- 189 trial. $^{10}$ All patients were assumed to receive end-of-life care before death.

Each health state was assigned a health utility from published studies. Only direct costs were considered and adapted for 2018 US dollars using the Medical Care Consumer Price Index. All costs and health outcomes were discounted at an annual discount rate of $3 \% .{ }^{11}$ The model simulated a 20-year period and each model cycle represented 21 days because in the clinical trial patients received pembrolizumab plus chemotherapy every 3 weeks. ${ }^{10}$ The primary outputs of the Markov model included cost and quality-adjusted life years (QALYs), which were applied to estimate the incremental costeffectiveness ratio (ICER). All analyses were performed in TreeAge pro 2018 software (https://www.treeage.com).

\section{Model probabilities}

The probability of transition of disease progression and from any state to death were from the survival curve of pembrolizumab or placebo combined with chemotherapy in the keynote-189 trial. ${ }^{10}$ We used the GetData Graph Digitizer software (V.2.25) to extract the data points of the Kaplan-Meier curves. According to the Akaike information criterion (AIC) and Bayesian information criterion (BIC), the PFS data points were fitted by a Weibull distribution, and overall survival (OS) data points were fitted with an exponential distribution. ${ }^{12}$ The distribution parameters were calculated using the method of Hoyle et al. ${ }^{12}$ Finally, the PFS and OS rates of each cycle were estimated by $\exp \left(-\lambda \mathrm{t}^{\gamma}\right)$ and $\exp (-\lambda \mathrm{t})$, respectively, where $\lambda$ is the scale parameter, $\gamma$ is the shape parameter and $t$ is survival time (table 1 near here).

Costs

Only direct costs, including the costs of the drug, premedication, administration and management of serious adverse events (AEs) (table 1 near here), were considered in our evaluation. In the PFS state, the cost of the intravenous drug for 3-week cycle was based on the following doses: pembrolizumab $200 \mathrm{mg} /$ cycle, pemetrexed $500 \mathrm{mg} / \mathrm{m}^{2}$, cisplatin $75 \mathrm{mg} / \mathrm{m}^{2}$ and carboplatin $400 \mathrm{mg} / \mathrm{m}^{2}$.

The model considered the hospitalisation cost of patients with $\mathrm{AE} \geqq$ grade 3 , and the incidence rate exceeded $5 \%$ because these AEs were of great concern to clinicians. ${ }^{13}$ And then the incidence rates of neutropenia and pneumonia from the Keynote-189 trial were used to calculate the cost of AEs treatments. ${ }^{10}$

Based on the Keynote- 189 trial, ${ }^{10} 30.5 \%$ of the patients in the pembrolizumab plus chemotherapy group and $46.6 \%$ in the placebo plus chemotherapy group received subsequent therapy after disease progression. Among patients in the pembrolizumab plus chemotherapy group, 3\% received carboplatin plus pemetrexed, 2.3\% received docetaxel plus ramucirumab, $17.8 \%$ received docetaxel, $4 \%$ received nivolumab and $3.4 \%$ received continuation maintenance treatment of pembrolizumab; among patients in the placebo plus chemotherapy group, $1.7 \%$ received docetaxel, $7.8 \%$ received nivolumab and $38 \%$ received crossover treatment with pembrolizumab. Patients who died accrued the cost of terminal care, including hospitalisation, palliative chemotherapy, doctor consultation, laboratory and diagnostic tests, according to the published literature. ${ }^{14}$

The mean value of a body surface area and body weight are $1.84 \mathrm{~m}^{2}$ and $82 \mathrm{~kg}$, respectively. ${ }^{13} 15$ The drug costs were taken from the Centers for Medicare and Medicaid Services. ${ }^{16}$ Administration costs were calculated according to the Medicare physician fee schedule for $2018 .{ }^{17}$ The costs of AEs and end-of-life care were derived from the published literature. ${ }^{13}$

\section{Outcome measures}

The outcome indicator of the study was QALYs, which is defined by the patient's life years and health utility. In accordance with the approach of Anna Oh $e t a l,{ }^{18}$ we also considered the disutility of AE. Baseline utility and disutility values were referenced in the published literature (table 1 near here). ${ }^{19} 20$

\section{Analysis}

The uncertainty of the parameters was evaluated by oneway sensitivity analysis and probabilistic sensitivity analysis, through the use of tornado diagrams and Monte Carlo simulation, respectively. The beta distribution was applied for the utilities, and the lognormal distribution was applied for the cost. Utilities were varied over their 95\% CIs. In general, the upper and lower limits of the 
Table 1 Parameters for cost effectiveness model

\begin{tabular}{|c|c|c|c|c|c|}
\hline \multirow[b]{2}{*}{ Parameter } & \multicolumn{2}{|c|}{ Pembrolizumab } & \multicolumn{2}{|l|}{ Placebo } & \multirow[b]{2}{*}{ Distribution } \\
\hline & Value & Ranges & Value & Ranges & \\
\hline \multicolumn{6}{|l|}{ Probabilities } \\
\hline \multicolumn{6}{|l|}{ PFS (Weibull) } \\
\hline Scale $(\lambda)$ & \multicolumn{2}{|l|}{0.0448} & \multicolumn{2}{|l|}{0.0876} & \\
\hline Shape $(\gamma)$ & \multicolumn{2}{|l|}{1.2675} & \multicolumn{2}{|l|}{1.2312} & \\
\hline \multicolumn{6}{|l|}{ OS(exponential) } \\
\hline Scale $(\lambda)$ & \multicolumn{2}{|l|}{0.0290} & \multicolumn{2}{|l|}{0.0586} & \\
\hline \multicolumn{6}{|l|}{ Costs $(\$)$} \\
\hline Pembrolizumab/mg ${ }^{16}$ & 48.57 & $+/-25 \%$ & 48.57 & $+/-25 \%$ & Lognorm \\
\hline Pemetrexed $/ \mathrm{mg}^{16}$ & 6.75 & $+/-25 \%$ & 6.75 & $+/-25 \%$ & Lognorm \\
\hline Cisplatin $/ \mathrm{mg}^{16}$ & 0.20 & $+/-25 \%$ & 0.20 & $+/-25 \%$ & Lognorm \\
\hline Carboplatin $/ \mathrm{mg}^{16}$ & 0.06 & $+/-25 \%$ & 0.06 & $+/-25 \%$ & Lognorm \\
\hline Chemotherapy infusion 1 hour $^{16}$ & 145 & $+/-25 \%$ & 145 & $+/-25 \%$ & Lognorm \\
\hline Chemotherapy infusion additional hour ${ }^{16}$ & 32 & $+/-25 \%$ & 32 & $+/-25 \%$ & Lognorm \\
\hline Subsequent therapies/cycle ${ }^{16}$ & 1160 & $+/-25 \%$ & 4394 & $+/-25 \%$ & Lognorm \\
\hline End-of-life care ${ }^{14}$ & 33009 & $+/-25 \%$ & 33009 & $+/-25 \%$ & Lognorm \\
\hline AE hospitalisation cost ${ }^{13}$ & 3538 & $+/-50 \%$ & 3005 & $+/-50 \%$ & Lognorm \\
\hline \multicolumn{6}{|l|}{ Baseline utilities } \\
\hline $\mathrm{PFS}^{19}$ & 0.71 & $0.67-0.76$ & 0.71 & $0.67-0.76$ & Beta \\
\hline disease progression survival ${ }^{19}$ & 0.67 & $0.59-0.75$ & 0.67 & $0.59-0.75$ & Beta \\
\hline \multicolumn{6}{|l|}{ Disutilities } \\
\hline Neutropenia $a^{20}$ & 0.09 & $0.060-0.119$ & 0.09 & $0.060-0.119$ & Beta \\
\hline Pneumonia ${ }^{20}$ & 0.09 & $0.059-0.121$ & 0.09 & $0.059-0.121$ & Beta \\
\hline
\end{tabular}

$A E$, adverse effect; OS, overall survival;PFS, progression-free survival.

parameters were taken from the literature, and if otherwise, upper and lower limits of $25 \%$ were set. All baseline values and ranges for variables are shown in table 1 .

\section{Patient and public involvement}

No patients or public were involved in the study.

\section{RESULTS}

\section{Base case analysis}

Weibull and exponential models used to fit the survival curves from the clinical trial (online supplementary appendix 1), which show that the decision analysis model established in this study can reflect the clinical effects very well. In the base case analysis, the lifetime cost of using pembrolizumab plus chemotherapy was $\$ 288532$ compared with $\$ 137123$ for placebo plus chemotherapy. When considering effectiveness, the pembrolizumab plus chemotherapy strategy yielded 1.61 QALYs, compared with 0.83 QALYs for the placebo plus chemotherapy strategy. The ICER of pembrolizumab plus chemotherapy was calculated as \$194 372/QALY compared with the placebo plus chemotherapy. When pembrolizumab cost $\$ 12.05$ and $\$ 31.38 / \mathrm{mg}$, the ICERs approximated the
WTP thresholds of $\$ 100000$ and $\$ 150$ 000/QALY, respectively (table 2 ).

\section{Sensitivity analysis}

The tornado diagrams present the results of one-way sensitivity analyses. Obviously, the cost of pembrolizumab, the cost of subsequent treatment in the placebo-combination group and baseline utility values of OS were the most relatively sensitive parameters, and the ICER range was

Table 2 Pembrolizumab plus chemotherapy costeffectiveness at additional modelled price points

\begin{tabular}{lcc}
\hline Parameter & \multicolumn{2}{c}{ Base case model analysis* } \\
\hline WTP value, \$/QALY & 100000 & 15000 \\
\hline Nivolumab cost, \$/mg & 12.05 & 31.38 \\
Total cost, \$ & 176197 & 235651 \\
QALYs & 1.61 & 1.61 \\
ICER, \$/QALY & 99915 & 149907 \\
\hline
\end{tabular}

${ }^{*}$ Only the cost of pembrolizumab was varied.

ICER, incremental cost-effectiveness ratio; QALY, quality-adjusted life-year; WTP, Willingness-to-pay. 


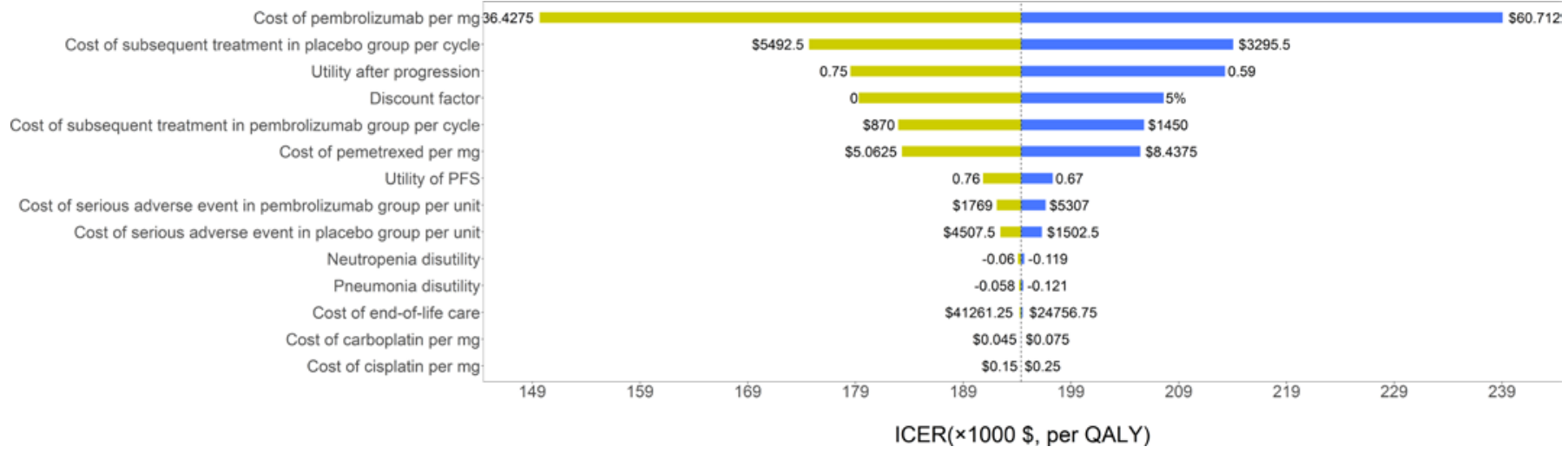

Figure 2 Tornado diagrams. The graphic shows the impact of varying individual model inputs on the cost-effectiveness of pembrolizumab plus chemotherapy for metastatic NSCLC. ICER, incremental cost-effectiveness ratio; nSCLC, non-small cell lung cancer.

from $\$ 149680 /$ QALY to $\$ 239065 /$ QALY (figure 2). The discount rate, the cost of subsequent treatment in the pembrolizumab-combination group, the cost of pemetrexed, the baseline utility value of PFS and the cost of AE management had little impact on the model.

The cost-effectiveness acceptability curve shows the probabilistic sensitivity analysis results of different willingness-to-pay (WTP) thresholds (figure 3). The probability that pembrolizumab combined with chemotherapy is cost-effective increased as WTP increased. The results showed that the probability of pembrolizumab plus chemotherapy being cost-effective was $0 \%$ at a WTP threshold of $\$ 130000 /$ QALY. If WTP threshold is $\$ 192$ 000/QALY, the pembrolizumab plus chemotherapy strategy show a 50\% chance cost-effectiveness (figure 3).

The results of the subgroup analysis showed that pembrolizumab combined with chemotherapy was the most cost-effective $(36 \%)$ for patients who had never smoked at a WTP threshold of $\$ 100000$. When the WTP threshold was $\$ 150000$, the probability of pembrolizumab combined with chemotherapy being cost-effective in the

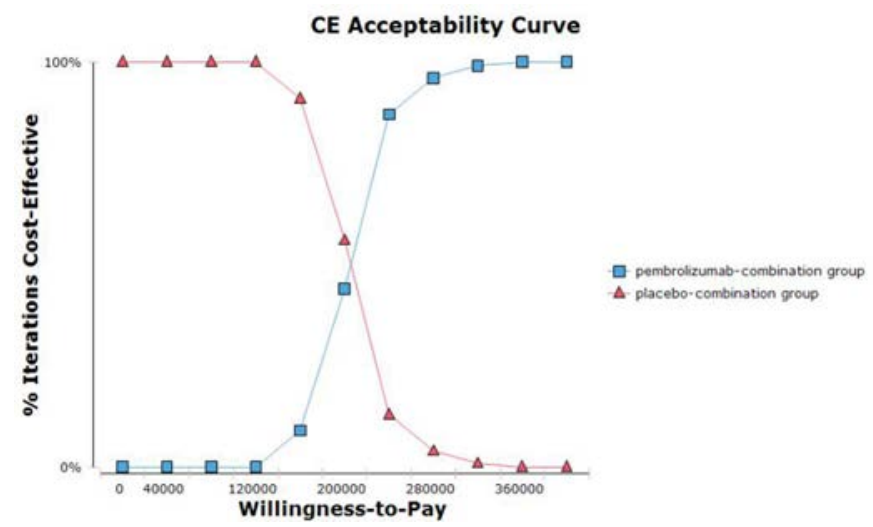

Figure 3 Cost-effectiveness (CE) acceptability curve. This plot represents the results of a probabilistic sensitivity analysis (for details, see Methods) comparing the costeffectiveness of pembrolizumab-pemetrexed-platinum versus placebo-pemetrexed-platinum in metastaticnon-small cell lung cancer (NSCLC). subgroup of never-smoking and female patients was $100 \%$ (see online supplementary appendix 2).

\section{DISCUSSION}

We performed a cost-effectiveness analysis of pembrolizumab in addition to chemotherapy in previously untreated metastatic NSCLC. Based on our model, the ICER for pembrolizumab plus chemotherapy was estimated as \$194 372/QALY compared with the placebo plus chemotherapy. The results showed that the probability of pembrolizumab plus chemotherapy being costeffective was $0 \%$ at a WTP threshold of $\$ 130$ 000/QALY.

There are many other studies that have analysed the cost-effectiveness of pembrolizumab for advanced NSCLC in different setting. ${ }^{13} 14$ 21-24 In the KEYNOTE-024 trial, pembrolizumab demonstrated the incremental survival benefits and better safety profile versus chemotherapy for first-line treatment of PD-L1 -positive $(\geq 50 \%)$ metastatic NSCLC patients, ${ }^{25}$ Based on the KEYNOTE-024 trial, a US-based study found that pembrolizumab was costeffective, with an ICER of $\$ 97621 / \mathrm{QALY}^{14}$ a study by Georgieva et al demonstrated that pembrolizumab monotherapy was cost-effective in the USA but not the UK, ${ }^{24}$ a study by $\mathrm{Hu}$ et al conducted in the UK demonstrated that pembrolizumab plus chemotherapy was not costeffective, with an ICER of $£ 86913 / \mathrm{QALY}^{23}$ and a French study found that pembrolizumab appears cost-effective. ${ }^{22}$ Our results differ from the above results may be due to different health systems and costs in different countries, which leads to different cost-effectiveness conclusions. Based on the KEYNOTE-010 trial, a study analysed the cost-effectiveness of pembrolizumab and docetaxel as second-line treatment for PD-L1 positive advanced NSCLC from the US third-party payer perspective. The results showed that the ICER was $\$ 168$ 619/QALY, which was cost-effective at a threshold of three times GDP per capita $(\$ 171660) .{ }^{13}$ These data provide reference value for evaluating the total cost of therapy and the value of regimens for advanced NSCLC. 
Our one-way sensitivity analysis revealed that the cost of pembrolizumab had a great influence on the results of the study. High drug prices are the result of the monopoly of pharmaceutical companies and restrictions on the negotiating power of the payer. ${ }^{26}$ This can be addressed by providing more meaningful price negotiation opportunities for payers and providing more evidence of a cost-effectiveness comparison of treatment regimens. ${ }^{26}$ We can also reduce the cost of administration by using personalised dosing. Recent study has shown that personalised dosing $(2 \mathrm{mg} / \mathrm{kg})$ and fixed dosing $(200 \mathrm{mg})$ of pembrolizumab have equivalent efficacy. ${ }^{27}$ Avoiding drug waste is extremely important in an era of valuebased cancer therapy. ${ }^{27}$ When our study used $2 \mathrm{mg} / \mathrm{kg}$ of pembrolizumab based on the average weight of $82 \mathrm{~kg}$, ${ }^{15}$ the ICER was reduced to $\$ 171751$. We believe that manufacturers are responsible for providing multiple sizes of vials to minimise the chance of wastage.

However, there are few limitations to our study that deserve consideration. First, we used cost parameters provided by Medicare, which may be lower than private insurers. ${ }^{28}$ Second, the health utility values were taken from other data sources instead of patients who participated in the Keynote 189 trial, which limits the accuracy of our results. Unfortunately, the clinical trial did not report the quality of life. Third, our analysis did not estimate the costs for all AEs in the PFS state, which may lead to underestimation of AEs costs. However, considering the low incidence, we expect the inclusion of all AEs would not change the conclusions of the present analysis. In addition, our model applied sensitivity analysis to a wide variation of these parameters, and it does not affect the results. Fourth, our analysis was based on the Keynote 189 trial, which excluded patients with sensitising EGFR or ALK translocation, because they usually used targeted agents as first-line treatment. However in the real-world setting, these patients with unknown EGFR or ALK translocation were also likely to be received PD-L1 testing and treated with pembrolizumab. Finally, our study directly compared pembrolizumab plus chemotherapy with chemotherapy according to the KEYNOTE-189 trial. Although there are other potential first-line treatments for advanced non-small cell lung cancer, our study did not indirectly compare them because of the lack of convincing trial data and robust head-to-head trial data.

Overall, the combination of pembrolizumab and chemotherapy for patients with metastatic NSCLC that we studied has high incremental cost and modest incremental benefit. New treatment technology for tumour is continuously undergoing development, but the price of tumour drugs is also rising dramatically. Based on our analysis, pembrolizumab offers lower value at current cost. The provision of cost-effective care requires new pricing and payment systems to support. The process for approving new drugs and the process of incorporating them into the guidelines must balance costs and benefits, and our research can offer decision-making information for this purpose.
Contributors Study concepts and design: CT; Clinical program: FM; Analysis and interpretation of the data: LP, YP, QL; The drafting and revising of the paper: $X Z$ and XW; Final approved of manuscript: All authors; all authors agree to be accountable for all aspects of the work.

Funding This work was supported by the National Natural Science Foundation of China [grant numbers 81401547, 81603081]; and the Key Science-Technology Research and Development Program of Hunan Province [grant number 2016JC2062].

Competing interests None declared.

Patient consent for publication Not required.

Provenance and peer review Not commissioned; externally peer reviewed.

Data availability statement Data are available upon reasonable request.

Open access This is an open access article distributed in accordance with the Creative Commons Attribution Non Commercial (CC BY-NC 4.0) license, which permits others to distribute, remix, adapt, build upon this work non-commercially, and license their derivative works on different terms, provided the original work is properly cited, appropriate credit is given, any changes made indicated, and the use is non-commercial. See: http://creativecommons.org/licenses/by-nc/4.0/.

\section{ORCID iD}

Chongqing Tan http://orcid.org/0000-0002-6007-4170

\section{REFERENCES}

1 WHO. International agency for Reseach on cancer. estimated number of new cases in 2018, world, both sexes, all age. Available: http://globocan.iarc.fr [Accessed 15 Dec 2018].

2 Nation Comprehensive Cancer Network. Non-Small cell lung cancer. 2nd edn, 2018. https://www.nccn.org/

3 Peters S, Kerr KM, Stahel R. PD-1 blockade in advanced NSCLC: a focus on pembrolizumab. Cancer Treat Rev 2018;62:39-49.

4 Weiss GJ. A new era of treating advanced lung cancer is upon us. Transl Lung Cancer Res 2018;7:S202-5.

5 Garon EB, Rizvi NA, Hui R, et al. Pembrolizumab for the treatment of non-small-cell lung cancer. N Engl J Med 2015;372:2018-28.

6 Scarpace SL. Metastatic squamous cell non-small-cell lung cancer (NSCLC): disrupting the drug treatment paradigm with immunotherapies. Drugs Context 2015;4:1-7.

7 Armand P. Immune checkpoint blockade in hematologic malignancies. Blood 2015;125:3393-400.

8 Dang TO, Ogunniyi A, Barbee MS, et al. Pembrolizumab for the treatment of PD-L1 positive advanced or metastatic non-small cell lung cancer. Expert Rev Anticancer Ther 2016;16:13-20.

9 Sul J, Blumenthal GM, Jiang X, et al. FDA approval summary: pembrolizumab for the treatment of patients with metastatic nonsmall cell lung cancer whose tumors express programmed DeathLigand 1. Oncologist 2016;21:643-50.

10 Gandhi L, Rodríguez-Abreu D, Gadgeel S, et al. Pembrolizumab plus chemotherapy in metastatic non-small-cell lung cancer. $N$ Engl $J$ Med 2018;378:2078-92.

11 Goldstein DA, Ahmad BB, Chen Q, et al. Cost-Effectiveness analysis of regorafenib for metastatic colorectal cancer. J Clin Oncol 2015;33:3727-32.

12 Hoyle MW, Henley W. Improved curve fits to summary survival data: application to economic evaluation of health technologies. BMC Med Res Methodol 2011;11:139.

13 Huang M, Lou Y, Pellissier J, et al. Cost-effectiveness of pembrolizumab versus docetaxel for the treatment of previously treated PD-L1 positive advanced NSCLC patients in the United States. J Med Econ 2017;20:140-50.

14 Huang M, Lou Y, Pellissier J, et al. Cost effectiveness of pembrolizumab vs. standard-of-care chemotherapy as first-line treatment for metastatic NSCLC that expresses high levels of PD-L1 in the United States. Pharmacoeconomics 2017;35:831-44.

15 Centers for Disease Control and Prevention. Faststats. Available: http://www.cdc.gov/nchs/fastats [Accessed 15 Dec 2018].

16 Center for Medicare and Medicaid services, 2018. Available: https:// www.cms.gov/apps/ama/license.asp?File=/Medicare/Medicare-Feefor-Service-Part-B-Drugs/McrPartBDrugAvgSalesPrice/Downloads/ 2018-July-ASP-Pricing-File.zip [Accessed 15 Dec 2018].

17 Goldstein DA, Chen Q, Ayer T, et al. First- and second-line bevacizumab in addition to chemotherapy for metastatic colorectal cancer: a United States-based cost-effectiveness analysis. J Clin Oncol 2015;33:1112-8. 
18 Oh A, Tran DM, McDowell LC, et al. Cost-Effectiveness of Nivolumab-Ipilimumab combination therapy compared with monotherapy for first-line treatment of metastatic melanoma in the United States. J Manag Care Spec Pharm 2017;23:653-64.

19 Chouaid C, Agulnik J, Goker E, et al. Health-related quality of life and utility in patients with advanced non-small-cell lung cancer: a prospective cross-sectional patient survey in a real-world setting. $J$ Thorac Oncol 2013;8:997-1003

20 Nafees B, Stafford M, Gavriel S, et al. Health state utilities for non small cell lung cancer. Health Qual Life Outcomes 2008:6:84.

21 Liao W, Huang J, Hutton D, et al. Cost-effectiveness analysis of firstline pembrolizumab treatment for PD-L1 positive, non-small cell lung cancer in China. J Med Econ 2019;1.

22 Chouaid C, Bensimon L, Clay E, et al. Cost-effectiveness analysis of pembrolizumab versus standard-of-care chemotherapy for firstline treatment of PD-L1 positive $(>50 \%)$ metastatic squamous and non-squamous non-small cell lung cancer in France. Lung Cancer 2019;127:44-52.
$23 \mathrm{Hu}$ X, Hay JW. First-Line pembrolizumab in PD-L1 positive nonsmall-cell lung cancer: a cost-effectiveness analysis from the UK health care perspective. Lung Cancer 2018;123:166-71.

24 Georgieva M, da Silveira Nogueira Lima JP, Aguiar P, et al. Costeffectiveness of pembrolizumab as first-line therapy for advanced non-small cell lung cancer. Lung Cancer 2018;124:248-54.

25 Reck M, Rodríguez-Abreu D, Robinson AG, et al. Pembrolizumab versus chemotherapy for PD-L1-positive non-small-cell lung cancer. N Engl J Med 2016;375:1823-33.

26 Kesselheim AS, Avorn J, Sarpatwari A. The high cost of prescription drugs in the United States: origins and prospects for reform. JAMA 2016;316:858-71.

27 Goldstein DA, Gordon N, Davidescu M, et al. A Phamacoeconomic analysis of personalized dosing vs fixed dosing of pembrolizumab in Firstline PD-L1-positive Non-Small cell lung cancer. J Natl Cancer Inst 2017;109.

28 Medicare Payment Advisory Commission. March 2018 report to the Congress: Medicare payment policy. Available: http://www.medpac. gov/-documents-/reports [Accessed 15 Dec 2018]. 